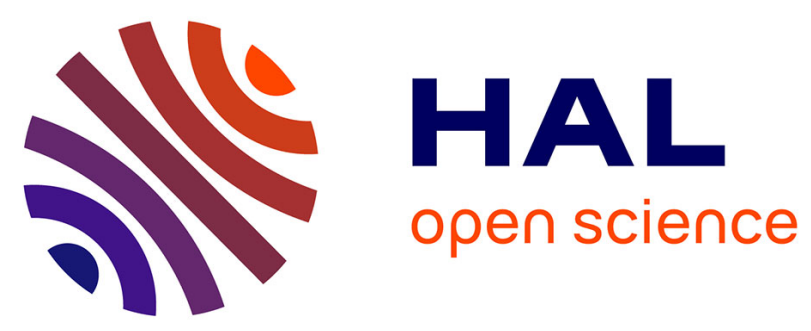

\title{
Développement cognitivo-émotionnel, régulation des émotions et comportements à risques: une étude exploratoire chez l'adolescent
}

Vincent Bréjard, Agnès Bonnet, Jean-Louis Pedinielli

\section{- To cite this version:}

Vincent Bréjard, Agnès Bonnet, Jean-Louis Pedinielli. Développement cognitivo-émotionnel, régulation des émotions et comportements à risques: une étude exploratoire chez l'adolescent. Neuropsychiatrie de l'Enfance et de l'Adolescence, 2005, 53 (8), pp.395-400. 10.1016/j.neurenf.2005.09.018 . hal-01314710

\section{HAL Id: hal-01314710 \\ https://hal-amu.archives-ouvertes.fr/hal-01314710}

Submitted on 22 Sep 2016

HAL is a multi-disciplinary open access archive for the deposit and dissemination of scientific research documents, whether they are published or not. The documents may come from teaching and research institutions in France or abroad, or from public or private research centers.
L'archive ouverte pluridisciplinaire HAL, est destinée au dépôt et à la diffusion de documents scientifiques de niveau recherche, publiés ou non, émanant des établissements d'enseignement et de recherche français ou étrangers, des laboratoires publics ou privés. 


\title{
Développement cognitivo-émotionnel, régulation des émotions et comportements à risques : une étude exploratoire chez l'adolescent
}

\author{
V. Bréjard*, A. Bonnet, J.-L. Pedinielli
}

* Laboratoire PsyCLE, UFR de psychologie, université de Provence. 29, avenue Robert-Schuman, 13621 Aix-en-Provence cedex 01, France

\section{Introduction}

Les comportements à risques chez les adolescents sont à l'origine d'une inquiétante morbidité, tant au niveau des conséquences sur leur intégrité physique que sur leur intégration scolaire et sociale, à moyen ou plus long terme. Si l'on remonte aux résultats de l'enquête nationale effectuée par l'INPES (Institut national pour l'éducation à la Santé) en 2000 [8], on observe les données suivantes : $21,9 \%$ des jeunes de 12 à 25 ans déclarent avoir eu au moins un accident ayant entraîné une consultation médicale ou une hospitalisation au cours des 12 derniers mois. En ce qui concerne le tabagisme, $31 \%$ des garçons et $29,6 \%$ des filles déclarent fumer régulièrement, et trois jeunes sur dix déclarent fumer tous les jours. L'alcool est consommé de manière régulière (une à plusieurs fois par semaine) par plus d'un garçon sur trois, et une fille sur six, cette consommation étant surtout importante le week-end (consommation " récréative »). Le cannabis est quant à lui consommé de manière répétée (dix fois dans l'année mais moins de dix fois par mois) ou régulière (au moins dix fois par mois) par 11, $9 \%$ des jeunes. Enfin, concernant la violence, $10,2 \%$ des garçons et 3,7 \% des filles déclarent avoir frappé quelqu'un au cours des 12 mois qui précédent l'enquête. Ces différents chiffres soulignent l'importance des comportements à risques et des troubles du comportement à cette période de la vie. De plus, chez certains adolescents, l'association entre ces comportements est régulièrement retrouvée. Dans une approche développementale, les conduites d'opposition, d'agression, et les autres conduites à risques proprement dites s'associent progressivement au cours du développement, constituant alors un véritable «syndrome de comportements à risques ». Si l'on se réfère à la Classification française des troubles mentaux de l'enfant et de l'adolescent Révisée-2000 [19], ces adolescents relèvent du chapitre 7 : « trouble des conduites et des comportements », et de la sous-section « autres troubles caractérisés des conduites » où sont classés des comportements répétitifs qui inquiètent et alertent l'entourage. Est évoquée la possibilité d'un trouble isolé ou s'inscrivant dans un tableau de conduites problématiques plus large. La CFMTEA R-2000 décrit ainsi la présence possible de manifestations associées telles que fugue (7.73), violences contre les personnes (7.74), conduites à risques (7.75), ou errances (7.76). Les caractéristiques psychopathologiques des adolescents présentant ces types de comportements sont maintenant bien connues : les descriptions rappellent l'intolérance à la frustration, la difficulté à ne pas répondre à une provocation par un comportement agressif, la difficulté à verbaliser des contenus psychiques. Sont également décrits la tonalité profon- dément anxieuse lors d'épisodes aigus, et un sentiment de vide [15]. Au-delà d'éléments contextuels, sociaux, ou de dimensions de personnalité $[16,18,23]$, un des éléments marquants du tableau clinique des adolescents engagés dans ces comportements répétitifs, est la difficulté qu'ils semblent rencontrer dans la capacité à se représenter et à communiquer les émotions ressenties face à des évènements de l'environnement. C'est pourquoi nous pensons qu'il est essentiel d'étudier le fonctionnement émotionnel de ces adolescents, afin de mieux comprendre certains des dysfonctionnements psychologiques qu'ils présentent et ainsi d'améliorer les techniques de prise en charge.

\section{Les dysfonctionnements émotionnels}

Le fonctionnement émotionnel a fait l'objet de différents travaux qui soulignent son implication dans les comportements à risques. Des facteurs tels que l'anhédonie [16], la propension à ressentir des émotions négatives (émotionnalité négative) [5] ont été retrouvés comme facteurs favorisant la tendance à s'engager dans des comportements à risque. Cependant, aucun travail à notre connaissance n'a étudié les relations entre l'alexithymie et les comportements à risques chez les adolescents.

\subsection{Les troubles de la conscience des émotions}

L'alexithymie, littéralement « absence de mots pour décrire les émotions ", est un concept proposé par Sifneos en premier lieu pour caractériser le fonctionnement mental de patients dits «psychosomatiques » [22]. Conçue en premier lieu comme explication étiopathogénique de certains troubles, l'alexithymie est actuellement considérée comme une dimension du fonctionnement mental pouvant aller du normal au pathologique $[7,20]$. Un certain nombre d'hypothèses théoriques ont été proposées pour tenter de rendre compte du fonctionnement alexithymique. L'une des plus intéressantes a été avancée en termes développementaux par Lane et Schwartz [12], à partir d'une intégration des théories de Piaget et de Werner. Elle considère que le fonctionnement émotionnel suit une ligne développementale hiérarchique, en une succession de niveaux de conscience émotionnelle. La conscience émotionnelle décrit la capacité d'un individu à faire l'expérience d'états subjectifs différenciés, à les interpréter correctement, ainsi qu'à les identifier et les imaginer chez autrui. Elle serait un type de traitement cognitif qui correspondrait à cinq niveaux de transformation structurale tout au long du développement : 
- la prise de conscience de sensations corporelles (avec le développement des premières capacités de symbolisation);

- la conscience des sensations dans l'action motrice (l'enfant prend conscience qu'il peut s'autostimuler) et de la possibilité de l'existence de sensations chez autrui ;

- la différenciation progressive d'émotions de base, proches des sensations perçues ;

- l'apparition de la capacité à discriminer des émotions complexes ;

- la capacité à ressentir, décrire chez soi-même et chez autrui des combinaisons d'émotions complexes et différenciées.

Ces cinq niveaux de conscience des émotions peuvent être considérés comme les équivalents des stades de développement de la pensée. Dans cette conception le fonctionnement alexithymique est ainsi considéré comme un arrêt à un stade précoce de la capacité à « reconnaître » ses propres états émotionnels [13]. Afin d'évaluer le niveau de conscience émotionnelle, une échelle a été développée, l'Échelle de Niveaux de Conscience Émotionnelle (Level of Emotional Awareness Scale ou LEAS) [11], qui a été traduite et validée en français [4].

\subsection{Les troubles thymiques dans les comportements à risques}

La présence d'éléments thymiques chez les adolescents qui s'engagent dans des comportements à risques a été retrouvée dans plusieurs études portant notamment sur la consommation abusive de substances psychoactives, la sexualité compulsive et non protégée avec des partenaires occasionnels, la conduite dangereuse de véhicules, ou encore les conduites agressives [9, $10,17,21]$. Parmi les différentes tentatives d'explication psychopathologiques, l'hypothèse d'un trouble thymique sous-jacent a été l'une des plus fréquemment évoquées. Pour Marcelli, les conduites à risques, particulièrement chez l'adolescent, s'inscrivent dans un contexte où la tonalité dépressive est un élément explicatif important, à côté d'autres variables telles que l'anxiété [15]. Cependant, cette relation entre trouble de l'humeur et comportements à risques n'est pas retrouvée dans toutes les études, et notamment celle de Flament et al. où la dépression était évaluée par un clinicien [6]. Outre, l'humeur dépressive, on retrouve fréquemment des troubles du fonctionnement cognitif, et notamment des cognitions dites « dysfonctionnelles ». Ces cognitions sont des représentations négatives du sujet sur lui-même ainsi que sur ses relations interpersonnelles; elles ont un effet négatif sur ses compétences à interagir avec autrui et l'environnement [3].

\section{Hypothèses}

Nous faisons l'hypothèse que les adolescents qui s'engagent dans des comportements à risques présentent un pattern cognitivo-émotionnel caractérisé par :

- un niveau de développement cognitif et émotionnel inférieur à ceux qui ne présentent pas de comportement à risques ;

- un niveau plus élevé d'alexithymie ;
- un niveau plus élevé de dépression ;

- un niveau plus important d'attitudes dysfonctionnelles.Ces différents éléments constituent des facteurs favorisant les comportements à risques.

\section{Méthodologie}

\section{1. Échantillon}

Notre échantillon est constitué de 56 sujets (sex-ratio $31 \mathrm{G} /$ $25 \mathrm{~F}$; âge moyen $=14,76 \pm 0,63$ ), issus de deux collèges de la région Provence-Alpes-Côte d'Azur. Les élèves étaient tous volontaires pour remplir les questionnaires, après autorisation des parents et des chefs d'établissement. Les passations étaient individuelles et se sont déroulées en présence des chercheurs qui ont ensuite expliqué les objectifs de la recherche. Les questionnaires ont été complétés en deux fois (en raison des capacités limitées de certains adolescents à maintenir un investissement dans la tâche pendant un temps suffisant). Nous avons ensuite constitué deux groupes de sujets par tirage au sort : 27 adolescents présentant des comportements à risques (groupe «CAR ») et 29 adolescents n'en présentant pas ou peu (groupe « NCAR »). Le critère d'inclusion dans le groupe CAR a été déterminé par le calcul de la médiane au questionnaire de comportement.

\subsection{Instruments d'évaluation}

Les comportements ont fait l'objet d'une double évaluation : par un entretien semi-directif au cours duquel les items suivants ont été abordés :

- consommation de substances psychoactives (tabac, cannabis, alcool, autre) ;

- conduites à risque (véhicule, sport, sexualité) ;

- conflit avec autrui (adultes, parents, pairs).

Nous avons ensuite croisé ces données avec celles recueillies auprès des enseignants et de l'équipe éducative (surveillants notamment). Les informations recueillies ont fait l'objet d'une cotation pour chaque item de 0 (comportement jamais présenté) à 4 (comportement très fréquent). Nous avons également utilisé les échelles suivantes :

- l'échelle de niveaux de conscience émotionnelle (LEAS) [4, 10]. Cette échelle est constituée de 20 items dans lesquels une situation d'interaction avec autrui est décrite. Le sujet doit répondre à deux questions pour chaque item : " que ressentirais-tu », « que ressentirait l'autre personne ? » La cotation s'effectue de 1 à 5 points en fonction de la qualité des réponses, et permet de calculer trois scores : un score global, un sous-score « soi » et un sous-score « autrui ». Nous avons été amenés, après un prétest, à adapter certains items de la manière suivante : remplacement de la forme « vous » par une forme «tu », et de « supérieur» dans les situations professionnelles par «professeur ». Les cota- 
tions ont été effectuées par les deux premiers auteurs en aveugle puis confrontées ;

- l'échelle d'alexithymie de Toronto (TAS-20) : cette échelle comporte 20 items de type Lickert en cinq points. Elle permet de calculer un score général et trois sous-scores : difficulté à identifier les émotions, difficultés à décrire les émotions, et pensée orientée vers l'extérieur. Dans cette étude, nous n'avons calculé que le score global. Les qualités métrologiques de cette échelle ont fait l'objet de nombreux travaux et ont permis de déterminer un score de 55 comme seuil au-delà duquel un individu est considéré comme alexithymique $[13,14]$;

- l'échelle d'attitudes dysfonctionnelles (DAS-A, Beck) est une échelle de type Lickert constituée de 40 items. Elle évalue la tendance à présenter des attitudes négatives envers soi-même, ainsi qu'à interpréter de manière erronée ses compétences sociales et personnelles. Ses qualités métrologiques sont satisfaisantes [2] ;

- l'inventaire abrégé de dépression de Beck (BDI) en 13 items a lui aussi été validé. Ses qualités psychométriques sont satisfaisantes [1].

\subsection{Analyses statistiques}

Nous avons effectué des analyses descriptives et multivariées que nous présenterons en trois étapes. Après avoir calculé les moyennes et écarts-types de chaque échantillon pour chaque variable, nous avons étudié l'effet de la variable type de groupe (CAR, NCAR) sur les variables niveau de conscience émotionnelle, alexithymie, attitudes dysfonctionnelles et dépression. Nous avons ensuite calculé les corrélations (coefficients de corrélations de Bravais-Pearson) entre les comportements à risques et les variables psychologiques mesurées. Enfin, nous avons estimé l'effet des variables émotionnelles dans les comportements à risques par un modèle de régression multiple (pas à pas ascendante), avec ajustement sur la variable sexe. Les analyses statistiques ont été effectuées avec le logiciel Statistica 6.0 ([c] Statsoft Inc, 2003).

\section{Résultats}

\subsection{Différences intergroupes}

Les résultats obtenus montrent que les adolescents qui présentent des comportements à risques ont un niveau de conscience émotionnelle inférieur aux adolescents qui n'en présentent pas $[F(1,54)=4,22, p=0,044]$. Cette différence est retrouvée logiquement à la dimension pour soi $[\mathrm{F}(1,54)$ $=10,28, \mathrm{p}=0,002]$ et à la dimension pour Autrui $[\mathrm{F}(1,54)$ $=11,36, \mathrm{p}=0,001]$. Ils ont un niveau d'alexithymie supérieur $[\mathrm{F}(1,54)=5,85, \mathrm{p}=0,018]$ et ont plus d'attitudes dysfonctionnelles $[F(1,54)=6,35, p=0,014]$. En revanche, on n'observe pas de différence significative entre les adolescents qui s'engagent dans des comportements à risques et ceux qui ne prennent pas de risques concernant l'intensité de l'humeur dépressive [F $(1,54)=3,84, p=0,055]$. Ce résultat est cependant à considé- rer avec précaution du fait de l'évaluation effectuée par un autoquestionnaire. Les scores moyens pour chaque groupe en fonction du sexe sont présentés dans le Tableau 1.

\subsection{Corrélations entre variables émotionnelles et comportements}

Des corrélations significatives entre dimensions psychologiques, symptômes dépressifs, fonctionnement cognitif et comportements à risques sont observées. L'échelle «Soi » de la LEAS est en corrélation négative avec la fréquence des comportements $(r=-0,54, p<0,05)$, de même que l'échelle LEAS «Autrui » $(\mathrm{r}=-0,52, \mathrm{p}<0,05)$. Concernant l'échelle générale de la LEAS, la corrélation est moins élevée, mais reste significative $(r=-0,38, p<0,05)$. Entre l'alexithymie et les comportements à risques, une relation positive est retrouvée $(r=0,36$, $\mathrm{p}<05$ ). Enfin, une relation positive est constatée entre les attitudes dysfonctionnelles présentées et les comportements à risques $(r=0,41, p<0,05)$. La corrélation n'est pas significative entre le niveau de dépression et les comportements $(r=-0,24$, $p>0,05$ ). L'ensemble des échelles ne présente pas de corrélations significatives entre elles. Lorsque l'on contrôle la variable sexe en calculant des corrélations partielles, les résultats retrouvés sont identiques. En outre, toujours lorsque l'on contrôle le sexe, la relation entre intensité dépressive et comportements à risques devient significative $(r=-0,39, \mathrm{p}<0,05)$.

5.3. Rôle des variables émotionnelles dans les comportements à risques

Le modèle de régression multiple testant la valeur prédictive des variables émotionnelles étudiées pour les comportements à risques est validé. Celui-ci explique une partie importante de la variance $\mathrm{du}$ nombre de comportements $\left(\mathrm{R}^{2}=0,3235\right)$. Il est statistiquement significatif $[F(4,51)=7,575, p=0,0001]$. Dans ce modèle, un faible niveau de conscience émotionnelle (bêta $=-0,276, p=0,02$ ) associé à l'alexithymie (bêta $=0,297$, $\mathrm{p}=0,011)$ et à un nombre élevé d'attitudes dysfonctionnelles (bêta $=0,337, \mathrm{p}=0,005$ ), constituent des facteurs favorisant les comportements à risques. La variable dépression est exclue du modèle de régression multiple. Le récapitulatif des résultats de l'analyse de régression multiple est présenté dans le Tableau 2.

\section{Discussion}

À l'analyse des différents résultats, nous constatons que trois de nos hypothèses peuvent être considérées comme validées, alors que deux autres ne le sont pas. La première portait sur le niveau de conscience émotionnelle : nous nous attendions à ce qu'il soit inférieur chez les adolescents du groupe « comportements à risques » (hypothèse 1). Cette hypothèse est validée. En revanche, la seconde hypothèse portant sur l'existence d'un fonctionnement alexithymique chez les adolescents «preneurs de risques » ne peut être considérée comme validée. Si l'on observe une différence significative entre les deux groupes (hypothèse 2), celle-ci doit être interprétée avec la plus 
Tableau 1

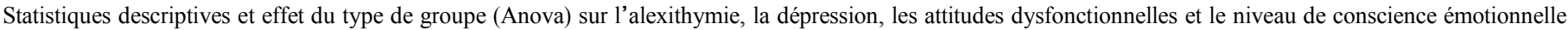

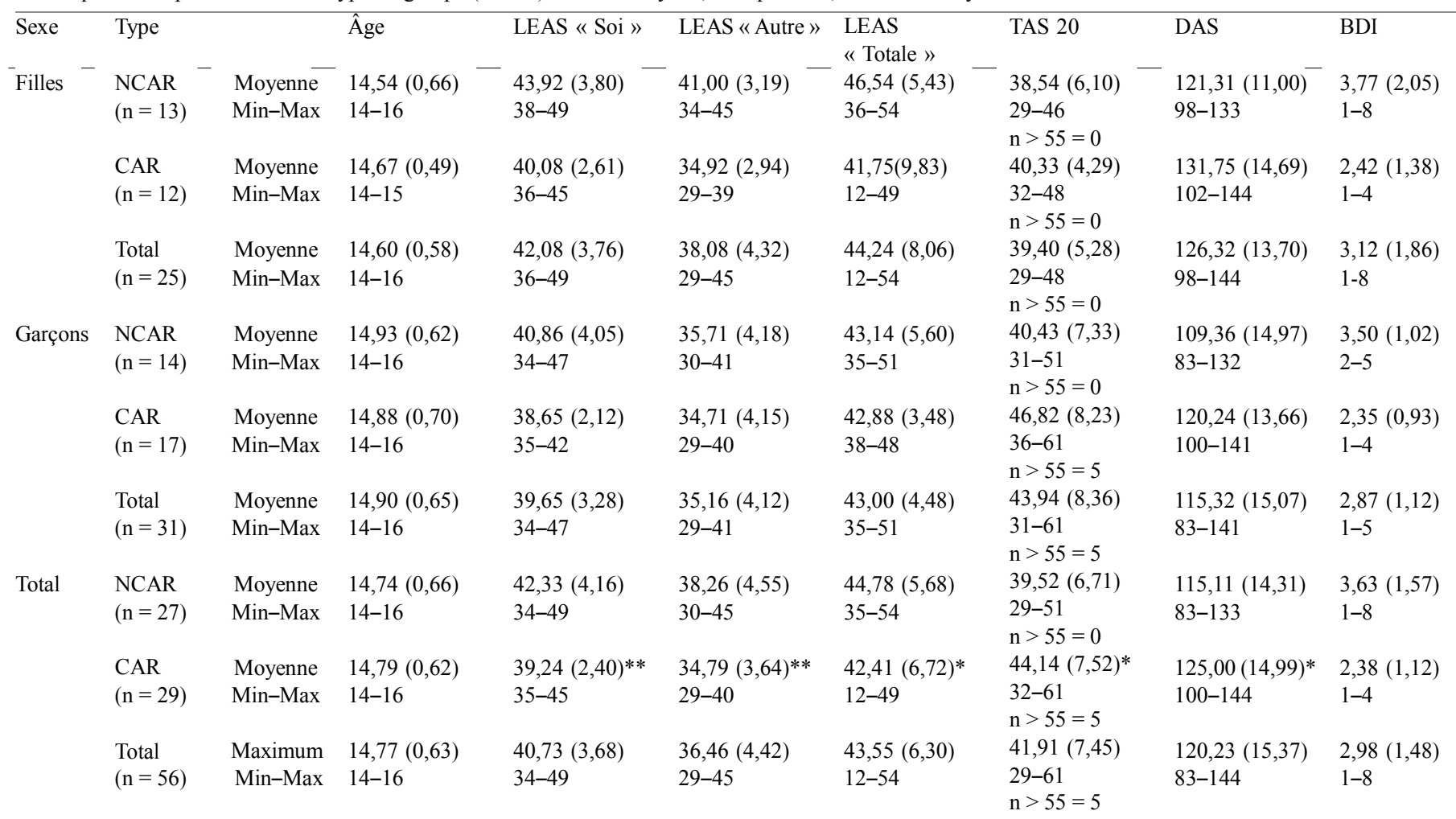

LEAS Soi : score à la sous-échelle pour soi de l'échelle de niveaux de conscience émotionnelle ; LEAS Autre : score à la sous-échelle pour autrui de l'échelle de niveaux de conscience émotionnelle ; LEAS Totale : score global à l'échelle de niveaux de conscience émotionnelle ; TAS-20 : score à l'échelle d'alexithymie de Toronto ; DAS : score à l'échelle d'attitudes dysfonctionnelles ; BDI : score à l'inventaire de dépression de Beck ; CAR : groupe d'adolescents présentant des comportements à risques ; NCAR : groupe d'adolescent sans comportements à risques ; ${ }^{*} \mathrm{p}<0,05,{ }^{* *} \mathrm{p}<0,01$.

Tableau 2

Récapitulatif du modèle explicatif des comportements à risque par le fonctionnement émotionnel et cognitif (régression multiple pas à pas), après ajustement sur le sexe

\begin{tabular}{|c|c|c|c|c|c|}
\hline & État & $\mathrm{t}$ & $\beta$ & $\mathrm{F}$ & $\mathrm{p}$ \\
\hline$\overline{\text { LEAS Totale }}$ & Incluse & $-2,4078$ & $-0,276 \overline{4}$ & 8,772 & $0,0196^{*}$ \\
\hline TAS-20 & Inclue & 2,6218 & 0,2969 & 6,874 & $0,0114 *$ \\
\hline DAS & Incluse & 2,9618 & 0,3376 & 5,797 & $0,0046^{* * *}$ \\
\hline \multirow[t]{2}{*}{ BDI } & Éliminée & $-1,4719$ & $-0,17$ & 2,1666 & 0,1471 \\
\hline & $\mathrm{R}^{2}=, 3235$ & $\mathrm{ddl}=4,51$ & & 7,5755 & $0,0001 * *$ \\
\hline
\end{tabular}

LEAS Totale : score global à l'échelle de niveaux de conscience émotionnelle ; TAS-20 : score à l'échelle d'alexithymie de Toronto ; DAS : score à l'échelle d'attitudes dysfonctionnelles ; BDI score à l'inventaire de dépression de Beck ; *p $<0,05, * * \mathrm{p}<0,01$

grande prudence, car les scores relevés sont inférieurs au seuil permettant de conclure à l'existence d'un fonctionnement alexithymique. La troisième hypothèse portait sur l'existence d'un niveau plus élevé de cognitions dysfonctionnelles chez les adolescents du groupe " comportements à risques » (hypothèse 3 ). Cette hypothèse est validée, les adolescents du groupe « comportements à risques » ayant plus d'attitudes dysfonctionnelles que les adolescents du groupe témoin. L'existence d'une tonalité dépressive chez les sujets "preneurs de risques» (hypothèse 4) n'est pas démontrée dans cette étude, ce qui pourrait s'expliquer selon nous par le choix méthodologique de l'autoévaluation. Des corrélations entre les variables psychologiques et les comportements à risques sont observées (excepté pour la dépression). Enfin, le modèle de régression multiple met en évidence que seules la conscience émotionnelle, l'alexithymie et les attitudes dysfonctionnelles sont impliquées dans les comportements à risques.

Ces résultats peuvent s'interpréter de deux manières. Les comportements à risques sont à l'origine de stimuli perceptifs internes par les sensations qu'ils procurent. Cependant, ces sensations ne peuvent être utilisées par l'adolescent pour tenter d'inscrire des traces cognitives de stimulations, du fait d'un faible niveau de conscience émotionnelle. Le sujet essaierait alors de répéter ces sensations correspondant à la reproduction d'éprouvés internes, en les utilisant comme éléments prototypiques d'émotions différenciées, et amener ainsi au développement d'un mécanisme proche d'une stratégie d'apprentissage. Faute d'éléments environnementaux lui permettant de se constituer une connaissance et une représentation mentale de ses états internes, ceux-ci demeurent au mieux répétitifs, au pire traumatiques. Ce dernier point nous amène à notre seconde interprétation. Les adolescents preneurs de risques, lorsqu'ils sont confrontés à des événements générateurs d'états émotionnels intolérables, seraient dans l'incapacité d'utiliser leurs émotions pour différer leurs réactions. En effet, les émotions permettent aux personnes d'orienter et de réguler leurs comportements. Or, cela suppose que l'émotion soit analysée, reconnue et interprétée. Chez les adolescents preneurs de risques, l'émotion semble mal différenciée (du fait d'un faible 
niveau de conscience émotionnel). Ce mode de fonctionnement mental provoquerait chez eux un risque de réponses comportementales inadaptées au contexte, du fait de l'incapacité à utiliser les émotions dans leur fonction de transaction avec l'environnement. Ces deux fonctionnalités seraient à l'origine d'une vulnérabilité émotionnelle, qui pourrait être renforcée par un niveau élevé d'attitudes dysfonctionnelles ne permettant pas une appréhension correcte de la réalité. Les attitudes dysfonctionnelles sont alors à considérer comme des conséquences possibles de la dépendance du sujet à l'égard de l'environnement, concernant des renforcements potentiels à la fois attendus et virtuellement menaçants.

Un élément dans nos résultats pourrait paraître en contradiction avec certains travaux antérieurs [16,20], mais concorde avec d'autres [6] : aucune dimension dépressive n'apparaît significativement dans notre groupe d'adolescents " preneurs de risques ». Outre les nécessaires limites inhérentes à l'autoévaluation de la dimension dépressive, un faible niveau de conscience émotionnelle a un effet péjoratif sur la capacité à se constituer des représentations mentales précises de ses états émotionnels et à les catégoriser. De ce fait, la dimension dépressive subjective évaluée ici a pu ne pas être mesurée par une autoévaluation. Cette dernière remarque nous amène à considérer les limites de notre étude : nos résultats sont en effet à considérer avec prudence. Premièrement en raison de la taille relativement modeste de notre échantillon qui de plus présente une surreprésentation féminine si l'on se réfère à la prévalence habituelle des comportements à risque plus importante chez les garçons. Deuxièmement, le caractère transversal de l'étude ne permet que de proposer des hypothèses sur l'articulation des variables étudiées. D'autres travaux, prospectifs, permettront de tester leur valeur prédictive pour les comportements à risques.

\section{Conclusion}

Ces différents constats pointent la nécessité, dans la prise en charge de ces adolescents, de tenir compte à la fois des dimensions cognitives, mais également des vulnérabilités qu'ils semblent présenter dans la capacité à supporter des éprouvés émotionnels déstabilisants. Cette perspective permettrait de proposer des modifications des techniques thérapeutiques en favorisant le passage d'une répétition vaine de ressentis proches des sensations physiques à un vécu non traumatique des éprouvés subjectifs.

\section{Références}

[1] Bourque P, Beaudette D. Étude psychométrique du questionnaire de dépression de Beck auprès d'un échantillon d'étudiants universitaires francophones. Can J Behav Sci 1982;14(3):211-8.

[2] Bouvard M, Cottraux J, Charles S, Cialdella P, Guerin J, Aimard G. Étude de validation sur une population française de l'échelle d'attitudes dysfonctinnelles de Weissman et Beck (DAS Forme A). J Ther Comp Cog 1994;4(4):127-35.
[3] Brown GP, Hammen CL, Craske MG, Wickens TD. Dimensions of dysfunctional attitudes as vulnerabilities to depressive symptoms. J Abnorm Psychol 1995;104(3):431-5.

[4] Bydlowski S, Corcos M, Paterniti S, Guilbaud O, Jeammet P, Consoli SM. Validation de la version française de l'échelle des niveaux de conscience émotionnelle. Encéphale 2002;28(4):310-20.

[5] Desrichard O, Denarié V. Sensation seeking and negative affectivity as predictors of risky behaviors: a distinction between occasional versus frequent risk-taking. Addict Behav 2005;30(7):1449-53.

[6] Flament MF, Cohen D, Choquet M, Jeammet P, Ledoux S. Phenomenology, psychosocial correlates, and treatment seeking in major depression and dysthymia of adolescence. J Am Acad Child Adolesc Psychiatry 2001;40(9):1070-8.

[7] Guilbaud O, Loas G, Corcos M, Speranza M, Stephan P, Perez-Diaz F, et al. L'alexithymie dans les conduites de dépendance et chez le sujet sain : valeur en population française et francophone. Ann Med Psychol (Paris) 2002;160(1):77-85.

[8] Guilbert P, Gauthier A, Baudier F, Trugeon A. Baromètre Santé 2000. Les comportements des 12-25 ans. Paris: INPES; 2004.

[9] King CA, Ghaziuddin N, McGovern L, Brand E, Hill E, Naylor M. Predictors of comorbid alcohol and substance abuse in depressed adolescents. J Am Acad Child Adolesc Psychiatry 1996;35(6):743-51.

[10] Kosunen E, Kaltiala-Heino R, Rimpela M, Laippala P. Risk-taking sexual behaviour and self-reported depression in middle adolescence-a schoolbased survey. Child Care Health Dev 2003;29(5):337-44.

[11] Lane RD, Quinlan DM, Schwartz GE, Walker PA, Zeitlin SB. The Levels of Emotional Awareness Scale: a cognitive-developmental measure of emotion. J Pers Assess 1990;55(1-2):124-34.

[12] Lane RD, Schwartz GE. Levels of emotional awareness: a cognitivedevelopmental theory and its application to psychopathology. Am J Psychiatry $1987 ; 144(2): 133-43$.

[13] Lane RD, Sechrest L, Riedel R, Shapiro DE, Kaszniak AW. Pervasive emotion recognition deficit common to alexithymia and the repressive coping style. Psychosom Med 2000;62(4):492-501.

[14] Loas G, Corcos M, Stephan P, Pellet J, Bizouard P, Venisse JL, et al. Factorial structure of the 20-item Toronto Alexithymia Scale: confirmatory factorial analyses in nonclinical and clinical samples. J Psychosom Res 2001;50(5):255-61.

[15] Marcelli D, Mezange F. Repeated accidents among adolescents. Anxiety traits, depressives and associated risk behavior. Rev Chir Orthop Reparatrice Appar Mot 1999;85(6):555-62.

[16] Michel G, Carton S, Jouvent R. Recherche de sensations et anhédonie dans les conduites de prise de risque. Étude d'une population de sauteurs a l'élastique (benji). Encéphale 1997;23(6):403-11.

[17] Michel G, Carton S, Perez-Diaz F, Mouren-Siméoni MC, Jouvent R. Symptomatologie dépressive et consommation de substances psychoactives à l'adolescence. Neuropsychiatr Enfance Adolesc 1999;48(10-11): 531-6.

[18] Michel G, Purper-Ouakil D, Mouren-Simeoni MC. Facteurs de risques des conduites de consommation de substances psychoactives à l'adolescence. Ann Med Psychol 2001;159(9):622-31.

[19] Mises R, Quemada N, Botbol M, Bursztejn C, Durand B, Garrabe J, et al. Une nouvelle édition de la classification française des troubles mentaux de l'enfant et de l'adolescent : la CFTMEA R-2000. Neuropsychiatr Enfance Adolesc 2002;50(4):233-61.

[20] Pedinielli JL. Psychosomatique et alexithymie. Paris: PUF; 1992.

[21] Pesa JA, Cowdery JE, Westerfield RC, Wang M. Self-reported depression and risk-taking behaviors among Hispanic adolescents. Psychol Rep 1997;81(1):235-43.

[22] Sifneos PE. The prevalence of "alexithymic" characteristics in psychosomatic patients. Psychother Psychosom 1973;22(2):255-62.

[23] Simantov E, Schoen C, Klein JD. Health-compromising behaviors: why do adolescents smoke or drink: identifying underlying risk and protective factors. Arch Pediatr Adolesc Med 2000;154(10):1025-33. 